\title{
Factors Contributing to Miss Appointments among 1-4 Years Old Toddlers for Routine Health Care in Government Health Clinics in Peninsular Malaysia
}

\author{
Noor Hafizan M $\mathrm{S}^{1}$, Rusnah $\mathrm{S}^{1}$, Khalib $\mathrm{L}^{1}$ \\ ${ }^{I}$ Department of Community Health, University Kebangsaan Malaysia Medical Centre, Cheras, Malaysia
}

\begin{abstract}
Scheduled routine health care follow up visits among 1-4 years old toddlers to government health clinics are not fully utilized and factors contributing to missed appointments are not well studied. Continuous health care among children under 5 years of age is one of the important factors to reduce morbidity and mortality. Therefore, this study aimed to identify the factors contributing to missed appointments among 1-4 years old toddlers for health care. Unmatched case control study among 570 mothers $(285$ cases and 285 controls) in five government health clinics in Tumpat district, Kelantan, Malaysia that serving a routine child health care was conducted using guided questionnaire. Analysis involved was binary logistic regression. Results showed that working mothers (adjusted OR:1.59, 95\% CI:1.10-2.30, $p:<0.05$ ), knowledge of the mothers about child health services in government health clinic (adjusted OR: 8.45, 95\% CI: 4.40-16.24, p:<0.001), mother's perception towards health facilities and services in government health clinic (adjusted OR: 1.59, 95\% CI: 1.102.30, p: $<0.05$ and mother's social support from people surrounding (adjusted OR : 4.89, 95\% CI: 2.40-9.98, $p:<0.001)$ were the factors contributing to missed appointments. The research findings indicate that special efforts should be made to help working mother, to promote child health care services in government health clinic and to increase social support to mother in child health care in improving their routine health care follow up visits to government health clinic.
\end{abstract}

Keywords Factors, government health clinic, miss appointment, toddler

\section{Introduction}

Regular health care among children in health facilities is very important for improvements of theirs health and become one of health intervention to reduce child mortality. The reported under five mortality rate (U5MR) has declined from 57 per 1,000 live births in 1970 via 16.6 in 1990 to 8.1 in 2005 (1). However, worldwide data in 2010 showed about 29,000 children under the age of five die every day, mainly from preventable causes (2). In order to make the mortality rate continuously reduced, the health of our children needs to observe closely by regular attendance to health facilities. In Malaysia, as part of the overall package of maternal and child health services in government health facilities, all children are needed to continue their regular attendance as scheduled for health care services (3). The availability of child health services in all government health facilities in Malaysia through the country's primary health care system has contributed to better health care for children and reduces children morbidity and mortality.

One of the challenges of this programme is a failure of continuous attendance at the recommended time or in easier term, misses appointments. Low rates of attendance among children especially 1-4 years old toddlers to government health clinic leave our young children at risk for undetectable medical problems (3). Many factors influencing the attendance of children for health care to health clinic. The factors include sosiodemografic factors like family size, household's income monthly, availability of transport, mother's education, mother's married status and age, children's age and sex, number of children, order of children, lack of knowledge or negative perception on child health services and facilities in health clinic include waiting time, attitude of service providers and limited accessibility to health care due to poor support (4 - 8). However, no research has been carried out to identify the factors that contributing to missed appointments among 1-4 years old toddlers to government health clinic in Malaysia, including in Kelantan where the rate of attendance is low. Hence, this study was conducted to identify the factors contributing to missed appointments among 1-4 years old toddlers for routine health care in government health clinics in Kelantan, Peninsular Malaysia.

\section{Materials And Methods}

Unmatched case control study was carried out over a period of four months (November 2011 to February 2012) in all 5 government health clinics in Tumpat district, Kelantan. Tumpat district is a coastal area in the North-eastern state of Kelantan, Peninsular Malaysia and is among the poorest district in the country. The study population was all children age from 1 to 4 years who registered in the government health clinics in Tumpat district for their regular health care services. Case group were mothers of 1-4 years old toddlers who 
missed follow up for 2 times and above. Control group were defined as mothers of 1-4 years olds toddler who never miss their follow up more than once. We reviewed the attendance status of the toddler using their clinic record from birth to their stated age.

The sample size was calculated based on two proportions comparison. It was determined using Fleiss (1981) formula with requirement for significance level $(\alpha)$ of 0.05 and $80 \%$ power (9). The ratio of cases and control were 1:1. The calculated sample size was 285 for each group. Therefore the total sample size was 570 (285 cases and 285 controls). The sampling was conducted using purposive sampling method. We collected the data through an interview with the mothers (respondents) of the 1-4 years old toddlers in health clinics, their house and also via telephone using a set of guided questionnaire. The questionnaire consisted of five parts that covered sociodemographic, knowledge, perception, practice and social support factors that related to health care of 1-4 years old toddlers in government health clinic in Malaysia. Prior to data collection, the questionnaire was pre-tested on 30 sample of similar population. The validation revealed good reliability with internal consistency of Cronbach's alpha 0.8 and good construct validity using factor analysis.

The collected data were entered into computer and analyzed using Statistical Package for Social Sciences (SPSS) for Windows Version 20.0 statistical software. The data was cleaned for inconsistencies and missing values. Descriptive, bivariate analysis and multivariate analysis were done. Simple frequency tables of maternal and child characteristics were made. The confidence level was set at $95 \%$. Factors that were found to be significant at $\mathrm{p}<0.05$ from the bivariate analysis were entered into a multiple logistic regression model. This study was approved by the National Medical Research Register (NMRR) and the ethical approval was obtained from National University of Malaysia Medical Research Ethical Committee, Cheras, Kuala Lumpur, Malaysia

\subsection{Respondent's demographic information}

\section{Results And Discussion}

A total of 570 respondents ( 285 cases and 285 controls group) responded to the survey. Table 1 shows the characteristics of the respondents. For the children's characteristics, no difference for age's group in case and control group. There were minimal differences for sex, number of sibling and order of the children in family for both groups. For the mother's characteristics, majority of them were married, attained primary/ secondary education level and aged 35 and above in both group. However, there were differences in working status in case and control group. Most of mothers were working (54.4\%) in case group compared to control group (43.9\%). There were more respondents with low monthly family income, having car and others transport and family size more than 5 persons in both group.

Table 1 Sociodemografic characteristics of the case and control group

\begin{tabular}{lrr}
\hline \multicolumn{1}{c}{ Sociodemografic factors } & $\begin{array}{c}\text { Case } \\
\mathbf{n}(\boldsymbol{\%})\end{array}$ & $\begin{array}{c}\text { Control } \\
\mathbf{n}(\boldsymbol{\%})\end{array}$ \\
\hline Gender of children: & $133(46.7)$ & $123(43.2)$ \\
Girl & $152(53.3)$ & $162(56.8)$ \\
Boy & & \\
Age of children: & $83(29.1)$ & $83(29.1)$ \\
$<2$ years old & $202(70.9)$ & $202(70.9)$ \\
$\geq 2$ years old & & \\
Number of siblings: & $202(70.9)$ & $211(74.0)$ \\
<5 person & $83(29.1)$ & $74(26.0)$ \\
$\geq 5$ person & & $82(28.8)$ \\
Birth order: & $68(23.9)$ & $203(71.2)$ \\
First & $217(76.1)$ & $10(3.5)$ \\
Others & & $275(96.5)$ \\
Status of mother: & $17(6.0)$ & \\
Widow & $268(94.0)$ & $101(35.4)$ \\
Married & & $184(64.6)$ \\
Age of mother: & $93(32.6)$ & $125(43.9)$ \\
< 35 years old & $192(67.4)$ & $160(56.1)$ \\
$\geq 35$ years old & & $226(79.3)$ \\
Employment status of mother: & $155(54.4)$ & \\
Employed & $130(45.6)$ & \\
Unemployed & $224(78.6)$ & \\
Education level of mother: & & \\
Low (Primary/secondary level) & & \\
& & \\
\hline
\end{tabular}


High (Tertiary level and above)

Transport:

Motorcycle only

Car and others

Household income:

Low ( $<$ RM1320)

High ( $\geq$ RM1320)

Family size:

$\leq 5$ person

$>5$ person
$61(21.4)$

$108(37.9)$

$177(62.1)$

$162(56.8)$

$123(43.2)$

$92(32.3)$

$193(67.7)$
59 (20.7)

$110(38.6)$

$175(61.4)$

$166(58.2)$

$119(41.8)$

$86(30.2)$

$199(69.8)$

\subsection{Statistical Analysis}

Table 2 shows simple binary logistic regression and multiple binary logistic regressions. In bivariate analysis (simple binary logistic regression), only employment status of the mother in sosiodemographic factors was found to be associated with missed appointments $(\mathrm{p}=0.012)$. The other independent variables like knowledge of mothers about child health care services in government health clinic $(p<0.001)$, perception of mothers toward facilities and services in government health clinic $(p<0.001)$ and social support to mother from people surrounding $(p<0.001)$ had significant association with missed appointments. All these independent variables were categorized by scoring it into 2 categories before bivariate analysis.

Multivariate analysis (multiple binary logistic regressions) was conducted to find the important determinants while adjusting for other factors. It was found; all the factors that significant in bivariate analysis retained it significant. Employed mothers were 2 times more likely to miss their 1-4 years old toddlers appointments for health care in government health clinic than unemployed mothers, [adjusted OR $=1.59,(95 \%$ CI:1.10-2.30)].

Mothers who had poor knowledge about child care services in government health clinic were 8 times more likely to miss their 1-4 years toddlers old appointments for health care in government health clinic than mothers who had good knowledge, [adjusted OR $=8.45$, (95\% CI: 4.40,16.24)]. Similarly, mothers who had negative perception towards facilities and services in government health clinic were 2 times more likely to miss their 1-4 years toddlers appointments for health care in government health clinic than mothers with positive perception, adjusted OR $=1.59$, (95\% CI:(1.10,2.30)]. Mothers who had poor social support were 5 times more likely to miss their 1-4 years toddlers appointments for health care in government health clinic than mother who had good social support, [adjusted OR $=4.89,(95 \%$ CI: 2.40,9.97)].

Table 2Factors associated with missed appointments among 1-4 years old toddlers for child health care in government health clinic

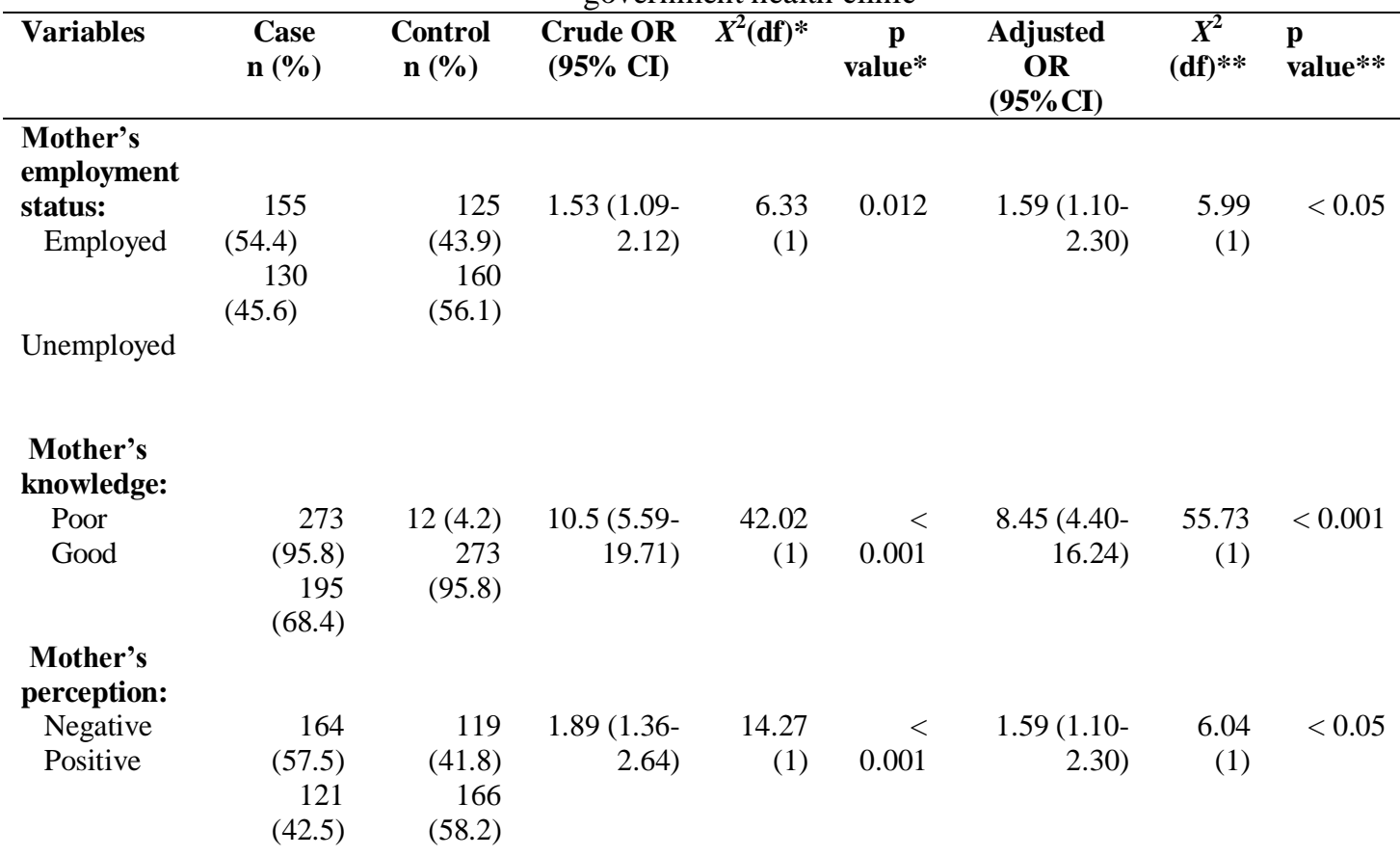


Factors Contributing to Miss Appointments among 1-4 Years Old Toddlers for Routine Health Care

$\begin{array}{crrrrrrrr}\text { support: } & 60(21.1) & 11(3.9) & 6.64(3.41- & 42.02 & < & 4.89(2.40- & 22.80 & <0.001 \\ \text { Poor } & 225 & 274 & 12.94) & (1) & 0.001 & 9.97) & (1) & \\ \text { Good } & (78.9) & (96.1) & & & & & \end{array}$

*Simple binary logistic regression, $* *$ multiple binary logistic regression

\subsection{Discussion}

Study has found employment status had significant association with missed appointments. Similar findings noted by Shamsul Azhar et al. (2012) (10). On the other hand, other sosiodemographic factors were not the factors that associated with missed appointments in this study, unlike reported by other studies (4, 7-8). Among the community in Tumpat district, transportation is not one of the barriers to go to government health clinic since it is situated near or in the community itself. Household income also was not contributing factors to missed appointments. This was in contrast with the findings of other studies (7-8). Possible reason for the insignificant finding may be due to services provided in government health clinic is free of charge. Other explanation to marital status of the mother is a cultural itself, whereby most of women in Kelantan state are active in outside business and may explain why to be a single mother is not a big problem to bring their children to clinic.

We found that education level of mother was not contributing to missed appointments. Possible reasons for the insignificant findings in this study might be due to the high number of respondents included in both groups were mother with education level up to secondary school that categorized in low education level may explain why the differences were not statistically significant. However study done by Shamsul Azhar et al. (2012) in Malaysia regarding missed appointments to government health clinic for immunization among children noted there was also no significant association between mother education level and missed appointments (10). Similar finding also noted by Shamsul Azhar et al. (2012) for age of the mother was not contributing to missed appointments (10).

Children characteristics like sex, gender, age, order in the family and number of siblings were not statistically significant factors for missed appointments in this study. These were in accordance with findings of Minty and Anderson (2004) and Getahun et al. (2010) (11, 12). However, Minty and Anderson (2004) revealed statistically significant association between number of siblings and attendance to health facilities (11). Possible reasons for the insignificant findings in this study might be due to the use of different research methodology (sample selection, data gathering and others).

Similar to previous studies, our findings indicate that poor knowledge among mother about child care services in government health clinic had significant association with missed appointments $(5,6,8,13)$. Odusanya et al. (2008) found mothers' knowledge of immunization and vaccination were significantly correlated with the rate of full immunization. Negative perception of mothers toward facilities and services in government health clinic had significant association with missed appointments. Similar finding was obtained from Tadesse et al. (2008) study which revealed mothers who had negative perception towards health institution were more likely to have defaulter children than mothers with positive perception. Poor social support to mother from people surrounding had significant association with missed appointments. Some other studies also found similar findings $(8,14)$.

\section{Conclusion}

On the basis of the findings of this research, we suggest that specific efforts are needed to target working women to assist them to adhere to government health clinics follow up as recommended. Poor knowledge on child health care services in government health clinic among mother was associated with missed appointments for child care to government health clinic. In recommending the above, we have to increase mother's knowledge on related knowledge of child health care via media, campaign and continuous health education in clinic or elsewhere. There are also barriers that may inhibit their use of the services due to poor social support to mother. The strengthening of social support networks for mother might be achieved through community group development and implementation of community systems that can provide them with the support they need when seeking health care. The conclusion of this study should have taken into consideration limiting factors such as the definition of case group which was limited to 1-4 years old toddlers who missed appointments for 2 times continuously due to difficulty in tracing the sample. These factors may have an influence on this study outcome. A limitation is that these data were from a purposive sample of 1-4 years old toddlers that registered for child health care in government health clinics in Tumpat district. Therefore, the findings pertain to the population of Tumpat district. We consider that the possible information bias was minimal, as parents were informed regarding the confidential of all information given. This finding also provides basic information for future research and the development of interventions to promote 1-4 years old toddlers adherence to government health clinic. 


\section{Acknowledgements}

We wish to thank Universiti Kebangsaan Malaysia for funding this study (project code: FF-331-2011).

\section{References}

[1] UNICEF Malaysia. State of World's Children. The United Nations Children's Fund, 2008.http://www.unicef.org/malaysia/msl/support_8072.html [1.9.10].

[2] WHO. Child health. World Health Organization, 2012. http://www.who.in

[3] MOH. Annual report 2008. Ministry of Health Malaysia, 2008.

[4] A.P.Alio and H.M. Salihu. Maternal Determinants of Pediatric Preventive Care Utilization among Blacks and Whites. Journal of the National Medical Association, 97(6), 2005.

[5] H. Tahirovic and A. Toromanovic. Glycemic control in diabetic children: role of mother's knowledge and socioeconomic status. European Journal of Paediatrics 169, 2010, 961-964.

[6] E. Borras, A. Dominguez, M. Fuentes, J. Batalla, N. Cardenosa and A. Plasencia. Parental knowledge of paediatric vaccination. BMC Public Health, 9, 2009.154.

[7] A. Ruhul, M. Nirali and S. Becker. Socioeconomic factors differentiating maternal and child health-seeking behavior in rural Bangladesh: A cross-sectional analysis. International Journal for Equity in Health 9(9), 2010.

[8] H. Tadesse, A. Deribew and M. Woldie. Predictors of defaulting from completion of child immunization in south Ethiopia, May 2008 - A case control study. BMC Public Health, 9, 2009, 150.

[9] J. L. Fleiss, Statistical methods for rates and proportions (New York: John Wiley, 1981).

[10] S. Shamsul Azhar, K. Nirmal, S. Nazarudin, H. Rohaizat, A. Azimatun Noor and H. Rozita. Factors Influencing Childhood Immunization Defaulters in Sabah, Malaysia. The International Medical Journal Malaysia, 11(1), 2012.

[11] B. Minty and C. Anderson. Non-Attendance at Initial Out-Patient Appointments at a Hospital-Based Child Psychiatric Clinic. Clin Child Psychol Psychiatry, 9, 2004, 403.

[12] A. Getahun, K. Deribe and A. Deribew. Determinants of delay in malaria treatment-seeking behaviour for under-five children in south-west Ethiopia: a case control study. Malaria Journal 9, 2010,320.

[13] O.O. Odusanya, E. F. Alufohai, F. P. Meurice and V. I. Ahonkhai. Determinants of vaccination coverage in rural Nigeria. BMC Public Health, 8, 2008, 381.

[14] M.E. Rutherford, J.D. Dockerty, M. Jasseh, S.R.C. Howie, P. Herbison, D. J. Jeffries, M. Leach, W. Stevens, K. Mulholland, R.A. Adegbola, and P.C. Hill. Access to health care and mortality of children under 5 years of age in the Gambia: a case-control study. Bulletin of the World Health Organization 87, 2009, 216-224. 